Algebraic $\&$ Geometric $\mathcal{T}_{\text {opology }}$

Volume 5 (2005) 1655-1676

Published: 1 December 2005

ATG

\title{
The fundamental groups of subsets of closed surfaces inject into their first shape groups
}

\author{
HANSPETER FischeR \\ ANDREAS ZASTROW
}

\begin{abstract}
We show that for every subset $X$ of a closed surface $M^{2}$ and every $x_{0} \in X$, the natural homomorphism $\varphi: \pi_{1}\left(X, x_{0}\right) \rightarrow \check{\pi}_{1}\left(X, x_{0}\right)$, from the fundamental group to the first shape homotopy group, is injective. In particular, if $X \subsetneq M^{2}$ is a proper compact subset, then $\pi_{1}\left(X, x_{0}\right)$ is isomorphic to a subgroup of the limit of an inverse sequence of finitely generated free groups; it is therefore locally free, fully residually free and residually finite.
\end{abstract}

AMS Classification 55Q52, 55Q07, 57N05; 20E25, 20E26

Keywords Fundamental group, planar sets, subsets of closed surfaces, shape group, locally free, fully residually free

\section{Introduction}

Understanding the fundamental group of a locally complicated space can be a difficult endeavor. Indeed, it was shown only recently that the fundamental group of the Sierpiński carpet is not isomorphic to a subgroup of the fundamental group of the Hawaiian Earrings [7]. Therefore, properties of such groups are often deduced indirectly. For example, the natural homomorphism $\varphi: \pi_{1}\left(X, x_{0}\right) \rightarrow \check{\pi}_{1}\left(X, x_{0}\right)$, from the fundamental group of any 1-dimensional (Hausdorff) compactum $\left(X, x_{0}\right)$ to its first shape homotopy group, has been shown to be injective [8]. Consequently, the fundamental group of any 1-dimensional (metric) continuum is isomorphic to a subgroup of the limit of an inverse sequence of finitely generated free groups; a fact already proved in [6] and also in [1, Theorem 5.11]. Injectivity of the above homomorphism has also been established for certain fractal-like trees of manifolds, which need not be semilocally simply connected at any point [10].

In this article, we make this same approach available for all subsets of closed surfaces. Our main result, Theorem 5, states that, for every subset $X$ of a 
closed surface $M^{2}$ and every $x_{0} \in X$, the naturally induced homomorphism $\varphi: \pi_{1}\left(X, x_{0}\right) \rightarrow \check{\pi}_{1}\left(X, x_{0}\right)$ is injective. In particular, if $X \subsetneq M^{2}$ is a proper compact subset, then $\pi_{1}\left(X, x_{0}\right)$ is isomorphic to a subgroup of the limit of an inverse sequence of finitely generated free groups. Consequently, $\pi_{1}\left(X, x_{0}\right)$ is locally free, fully residually free and residually finite (Corollary 7 ).

Another helpful notion in studying fundamental groups of complicated spaces is that of being homotopically Hausdorff, which was introduced in [1]. Proposition 8 below shows that if $X$ is any space such that $\varphi: \pi_{1}\left(X, x_{0}\right) \rightarrow \check{\pi}_{1}\left(X, x_{0}\right)$ is injective, then $X$ is homotopically Hausdorff at $x_{0}$; but the converse does not hold, in general. Therefore, our main theorem improves upon [5. Theorem 3.4], which states that every subset of the plane is homotopically Hausdorff at every one of its points.

The structure of this article is as follows: Section 2 provides a brief review of the definition of the first shape homotopy group. In Section 3, we then proceed to prove the main theorem. Our proofs will use some special properties of subsets of the plane, which are exported into Section 4. Among them are two crucial tools from the thesis [19]: Theorem 15] and Theorem 17. Since these results are central to our main theorem, we include a complete proof of Theorem 15] and sketch a proof of Theorem 17 in the appendix.

Acknowledgements This research was supported by the Faculty Internal Grants Program of Ball State University as well as the University of Gdańsk (research grant numbers BW UG 5100-5-0233-2, 5100-5-0096-5). The authors wish to thank these institutions for their hospitality during their respective visits. The authors would also like to thank the referee for very helpful remarks.

\section{The first shape homotopy group}

We recall the definition of the first shape homotopy group of a pointed separable metric space $\left(X, x_{0}\right)$. For more details, see [15. Chapter II, §3.3, Chapter I, $\S 5.4$, and Appendix 1, §3].

Consider the collection $\mathcal{C}$ of all pairs $(\mathcal{U}, *)$, where $\mathcal{U}$ is a locally finite open cover of $X$, and $* \in \mathcal{U}$ is a designated member with $x_{0} \in *$. Then $\mathcal{C}$ is naturally directed by refinement. Denote by $(N(\mathcal{U}), *)$ a geometric realization of the pointed nerve of $\mathcal{U}$, that is, a geometric realization of the abstract simplicial complex $\left\{\Delta \mid \emptyset \neq \Delta \subseteq \mathcal{U}, \bigcap_{U \in \Delta} U \neq \emptyset\right\}$ with distinguished vertex $*$. For every $(\mathcal{U}, *),(\mathcal{V}, *) \in \mathcal{C}$ such that $(\mathcal{V}, *)$ refines $(\mathcal{U}, *)$, choose a pointed simplicial map $p_{\mathcal{U V}}:(N(\mathcal{V}), *) \rightarrow(N(\mathcal{U}), *)$ with the property that the vertex 
corresponding to an element $V \in \mathcal{V}$ gets mapped to a vertex corresponding to an element $U \in \mathcal{U}$ with $V \subseteq U$. (Any assignment on the vertices which is induced by the refinement property will extend linearly.) Then $p_{\mathcal{U V}}$ is unique up to pointed homotopy and we denote its pointed homotopy class by $\left[p_{\mathcal{U V}}\right]$. For each $(\mathcal{U}, *) \in \mathcal{C}$ choose a pointed map $p_{\mathcal{U}}:\left(X, x_{0}\right) \rightarrow(N(\mathcal{U}), *)$ such that $p_{\mathcal{U}}^{-1}(S t(U, N(\mathcal{U}))) \subseteq U$ for all $U \in \mathcal{U}$, where $S t(U, N(\mathcal{U}))$ denotes the open star of the vertex of $N(\mathcal{U})$ which corresponds to $U$. (For example, define $p_{\mathcal{U}}$ based on a partition of unity subordinated to $\mathcal{U}$.) Again, such a map $p_{\mathcal{U}}$ is unique up to pointed homotopy and we denote its pointed homotopy class by [ $\left.p_{\mathcal{U}}\right]$. Then $\left[p_{\mathcal{U V}} \circ p_{\mathcal{V}}\right]=\left[p_{\mathcal{U}}\right]$. The so-called (pointed) Čech expansion

$$
\left(X, x_{0}\right) \stackrel{\left(\left[p_{\mathcal{U}}\right]\right)}{\longrightarrow}\left((N(\mathcal{U}), *),\left[p_{\mathcal{U} \mathcal{V}}\right], \mathcal{C}\right)
$$

is an $\mathrm{HPol}_{*}$-expansion, so that we can define the first shape homotopy group of $X$, based at $x_{0}$, by

$$
\check{\pi}_{1}\left(X, x_{0}\right)=\lim _{\longleftarrow}\left(\pi_{1}(N(\mathcal{U}), *), p_{\mathcal{U V} \#}, \mathcal{C}\right) .
$$

Remark 1 In the event that $X$ is compact, other $\mathrm{HPol}_{*}$-expansions are possible. For any inverse sequence

$$
\left(X_{1}, x_{1}\right) \stackrel{f_{1,2}}{\longleftarrow}\left(X_{2}, x_{2}\right) \stackrel{f_{2,3}}{\longleftarrow}\left(X_{3}, x_{3}\right) \stackrel{f_{3,4}}{\longleftarrow} \cdots
$$

of pointed compact polyhedra such that

$$
\left(X, x_{0}\right)=\lim _{\longleftarrow}\left(\left(X_{i}, x_{i}\right), f_{i, i+1}\right),
$$

the inverse limit projection

$$
\left(X, x_{0}\right) \stackrel{\left(p_{i}\right)}{\longrightarrow}\left(\left(X_{i}, x_{i}\right), f_{i, i+1}\right)
$$

with $p_{i}=f_{i, i+1} \circ p_{i+1}$ for all $i$, is also an $\mathrm{HPol}_{*}$-expansion. Therefore,

$$
\check{\pi}_{1}\left(X, x_{0}\right)=\lim _{\longleftarrow}\left(\pi_{1}\left(X_{1}, x_{1}\right) \stackrel{f_{1,2 \#}}{\longleftarrow} \pi_{1}\left(X_{2}, x_{2}\right) \stackrel{f_{2,3 \#}}{\longleftarrow} \pi_{1}\left(X_{3}, x_{3}\right) \stackrel{f_{3,4 \#}}{\longleftarrow} \cdots\right) .
$$

For example, an admissible choice is any nested sequence $X_{1} \supseteq X_{2} \supseteq \cdots$ of compact polyhedra, whose intersection is $X$, where $f_{i, i+1}$ is inclusion and $x_{i}=x_{0}$ for all $i$. If, on the other hand, $X$ is an arbitrary topological space, a definition of $\check{\pi}_{1}\left(X, x_{0}\right)$ is based on normal coverings $(\mathcal{U}, *)$ of $X$, rather than locally finite ones [15].

Since the maps $p_{\mathcal{U}}$ induce homomorphisms $p_{\mathcal{U} \#}: \pi_{1}\left(X, x_{0}\right) \rightarrow \pi_{1}(N(\mathcal{U}), *)$ such that $p_{\mathcal{U} \#}=p_{\mathcal{U V} \#} \circ p_{\mathcal{V} \#}$, whenever $(\mathcal{V}, *)$ refines $(\mathcal{U}, *)$, we obtain an induced homomorphism

$$
\varphi: \pi_{1}\left(X, x_{0}\right) \rightarrow \check{\pi}_{1}\left(X, x_{0}\right)
$$

given by $\varphi([\alpha])=\left(\left[\alpha_{\mathcal{U}}\right]\right)$ where $\alpha_{\mathcal{U}}=p_{\mathcal{U}} \circ \alpha$; or, in the compact case of Remark 1] by $\varphi([\alpha])=\left(\left[\alpha_{1}\right],\left[\alpha_{2}\right],\left[\alpha_{3}\right], \ldots\right)$ where $\alpha_{i}=p_{i} \circ \alpha$. 


\section{The main theorem}

We first establish our result for subsets of the plane:

Theorem 2 Let $X \subseteq \mathbb{R}^{2}$ be any subset and $x_{0} \in X$. Then the natural homomorphism $\varphi: \pi_{1}\left(X, x_{0}\right) \rightarrow \check{\pi}_{1}\left(X, x_{0}\right)$ is injective.

Before we present the proof of Theorem 2, we would like to point out that it fails for subsets of $\mathbb{R}^{3}$; even for Peano continua in $\mathbb{R}^{3}$. This is illustrated in the following example, which is taken from [20, Example 0.12] and based on [11, pp. 185-186].

Example 3 Let $H$ be the Hawaiian Earrings. That is, let $H=\bigcup_{k=1}^{\infty} H_{k}$ where $H_{k}=\left\{(x, y) \in \mathbb{R}^{2} \mid x^{2}+(y-1 / k)^{2}=(1 / k)^{2}\right\}$. Denote by $C$ the cone on $H$, with distinguished point $x_{0}=(0,0)$ in its base $H$. Identify two identical copies of $C$ along their distinguished points within $\mathbb{R}^{3}$, and call the resulting space $X$. Then $\varphi: \pi_{1}\left(X, x_{0}\right) \rightarrow \check{\pi}_{1}\left(X, x_{0}\right)$ is not injective. Indeed, a loop $\alpha:\left(S^{1}, *\right) \rightarrow\left(X, x_{0}\right)$ which runs through every circle of the two bases exactly once, always alternating between the two copies of $C$, is not null-homotopic in $X$, but null-homotopic in every compact polyhedral approximation of $X$ in $\mathbb{R}^{3}$.

Remark 4 It is well known that the natural homomorphism of Theorem 2 is typically not surjective, even for planar Peano continua. Indeed, such is the case for the Hawaiian Earrings $\left(H, x_{0}\right)$ of Example 3. To see why, put $X_{i}=H_{1} \cup H_{2} \cup \cdots \cup H_{i}$, let $x_{0}=x_{i}=(0,0)$, and define $f_{i, i+1}: X_{i+1} \rightarrow X_{i}$ by $f_{i, i+1}(z)=x_{0}$ for $z \in H_{i+1}$ and $f_{i, i+1}(z)=z$ for $z \in X_{i+1} \backslash H_{i+1}$. Then $\left(H, x_{0}\right)$ is the limit of the inverse sequence $\left(\left(X_{i}, x_{i}\right), f_{i, i+1}\right)$. While this time $\varphi: \pi_{1}\left(H, x_{0}\right) \rightarrow \check{\pi}_{1}\left(H, x_{0}\right)$ is injective [8. Corollary 1.2], it is not surjective: let $l_{i}:\left(S^{1}, *\right) \rightarrow\left(H_{i}, x_{0}\right)$ be a fixed homeomorphism and, following an idea of Griffiths [12, p. 185], consider for each $i$ the element

$$
g_{i}=\left[l_{1}\right]\left[l_{1}\right]\left[l_{1}\right]^{-1}\left[l_{1}\right]^{-1}\left[l_{1}\right]\left[l_{2}\right]\left[l_{1}\right]^{-1}\left[l_{2}\right]^{-1}\left[l_{1}\right]\left[l_{3}\right]\left[l_{1}\right]^{-1}\left[l_{3}\right]^{-1} \cdots\left[l_{1}\right]\left[l_{i}\right]\left[l_{1}\right]^{-1}\left[l_{i}\right]^{-1}
$$

of $\pi_{1}\left(X_{i}, x_{i}\right)$. Then the sequence $\left(g_{i}\right)$ is an element of the group $\check{\pi}_{1}\left(H, x_{0}\right)$ which is clearly not in the image of $\varphi: \pi_{1}\left(H, x_{0}\right) \rightarrow \check{\pi}_{1}\left(H, x_{0}\right)$.

Proof of Theorem 2 Let $\alpha:\left(S^{1}, *\right) \rightarrow\left(X, x_{0}\right)$ be a map with the property that $[\alpha] \neq 1 \in \pi_{1}\left(X, x_{0}\right)$. We wish to show that $\varphi([\alpha]) \neq 1 \in \check{\pi}_{1}\left(X, x_{0}\right)$. To this end, define

$$
Y=\alpha\left(S^{1}\right) \cup \bigcup\left\{U \mid U \text { is a (path) component of } \mathbb{R}^{2} \backslash \alpha\left(S^{1}\right) \text { with } U \subseteq X\right\} .
$$


We now apply the results of Section 4, By Lemma 12, $Y$ is a Peano continuum. Since $x_{0} \in Y \subseteq X$, we have $[\alpha] \neq 1 \in \pi_{1}\left(Y, x_{0}\right)$. Let $L$ and $\left(L_{i}\right)_{i}$ be as in Theorem 15. applied to the planar Peano continuum $Y$ with designated point $x_{0}$, and arrange for $z_{i} \notin X$ for all $i$. Since the inclusion $\left(Y, x_{0}\right) \hookrightarrow\left(L, x_{0}\right)$ is a pointed homotopy equivalence, we have $[\alpha] \neq 1 \in \pi_{1}\left(L, x_{0}\right)$. By Theorem [17 there is an $n$ such that $[\alpha] \neq 1 \in \pi_{1}\left(L_{n}, x_{0}\right)$. Let $\left\{D_{1}, D_{2}, \ldots, D_{n-1}\right\}$ be the closures of the bounded components of $\mathbb{R}^{2} \backslash L_{n}$. Then $z_{i} \in$ (int $\left.D_{i}\right) \backslash X$ for all $1 \leqslant i \leqslant n-1$. Since $[\alpha] \neq 1 \in \pi_{1}\left(L_{n}, x_{0}\right)$, Lemma [13 implies that $\alpha$ is not null-homotopic in $\mathbb{R}^{2} \backslash\left\{z_{1}, z_{2}, \ldots, z_{n-1}\right\}$. Choose a finite open cover $\mathcal{V}$ of $\mathbb{R}^{2} \backslash\left\{z_{1}, z_{2}, \ldots, z_{n-1}\right\}$, such that $p_{\mathcal{V} \#}: \pi_{1}\left(\mathbb{R}^{2} \backslash\left\{z_{1}, z_{2}, \ldots, z_{n-1}\right\}, x_{0}\right) \rightarrow \pi_{1}(N(\mathcal{V}), *)$ is an isomorphism. Define $\mathcal{U}=\{V \cap X \mid V \in \mathcal{V}\}$. Then $\mathcal{U}$ is a finite open cover of $X$. Notice, that $N(\mathcal{U})$ is a subcomplex of $N(\mathcal{V})$, and that the two maps $\left.p_{\mathcal{V}}\right|_{X}: X \rightarrow N(\mathcal{V})$ and $p_{\mathcal{U}}: X \rightarrow N(\mathcal{U}) \subseteq N(\mathcal{V})$ are homotopic as maps into $N(\mathcal{V})$, because they are contiguous. (That is, for every $x \in X, p_{\mathcal{V}}(x)$ and $p_{\mathcal{U}}(x)$ lie in a common simplex of $N(\mathcal{V})$.) If $\varphi([\alpha])=1 \in \check{\pi}_{1}\left(X, x_{0}\right)$, then $p_{\mathcal{U} \#}([\alpha])=1 \in \pi_{1}(N(\mathcal{U}), *)$. This would imply that $p_{\mathcal{V} \#}([\alpha])=1 \in \pi_{1}(N(\mathcal{V}), *)$, which is not the case.

Theorem 5 Let $M^{2}$ be a closed surface and $X \subseteq M^{2}$ any subset. Then for every $x_{0} \in X$, the natural homomorphism $\varphi: \pi_{1}\left(X, x_{0}\right) \rightarrow \check{\pi}_{1}\left(X, x_{0}\right)$ is injective.

Proof We will argue by way of contradiction and assume, contrary to the assertion, that there is a continuous function $\alpha:\left(S^{1}, *\right) \rightarrow\left(X, x_{0}\right)$ with the property that $[\alpha] \neq 1 \in \pi_{1}\left(X, x_{0}\right)$ and $\varphi([\alpha])=1 \in \check{\pi}_{1}\left(X, x_{0}\right)$. Consider the following commutative diagram of naturally induced homomorphisms:

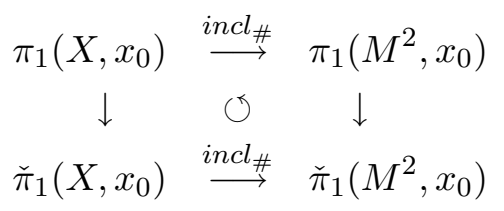

Since the righthand vertical homomorphism is an isomorphism, we conclude that $[\alpha]=1 \in \pi_{1}\left(M^{2}, x_{0}\right)$ and $X \neq M^{2}$. Replacing $M^{2}$, if necessary, we may therefore assume that $M^{2}$ has universal covering $q:\left(\mathbb{R}^{2}, \tilde{x}_{0}\right) \rightarrow\left(M^{2}, x_{0}\right)$ so that $\alpha$ lifts to a map $\tilde{\alpha}:\left(S^{1}, *\right) \rightarrow\left(\mathbb{R}^{2}, \tilde{x}_{0}\right)$ with $q \circ \tilde{\alpha}=\alpha$. Let us define $\bar{X}=q^{-1}(X)$. Then $\tilde{x}_{0} \in \bar{X} \subseteq \mathbb{R}^{2}$. Consider the natural homomorphism $\psi: \pi_{1}\left(\bar{X}, \tilde{x}_{0}\right) \rightarrow \check{\pi}_{1}\left(\bar{X}, \tilde{x}_{0}\right)$.

We claim that $\psi([\tilde{\alpha}])=1 \in \check{\pi}_{1}\left(\bar{X}, \tilde{x}_{0}\right)$. To verify this, let $\mathcal{U}$ be any cover of $\bar{X}$ by open sets of $\bar{X}$. Choose a sufficiently fine cover $\mathcal{V}$ of $\bar{X}$ by open sets of $\bar{X}$ such that (i) $\mathcal{V}$ refines $\mathcal{U}$; (ii) $\mathcal{C}=\{q(V) \mid V \in \mathcal{V}\}$ is a cover of $X$ by open sets 
of $X$; and (iii) there is a covering map $q^{\prime}$ (when restricted to path components of $N(\mathcal{V})$ ) in a commutative diagram:

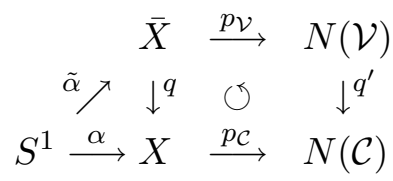

Since $\varphi([\alpha])=1 \in \check{\pi}_{1}\left(X, x_{0}\right)$, we have $p_{\mathcal{C} \#}([\alpha])=1 \in \pi_{1}(N(\mathcal{C}), *)$. Since $p_{\mathcal{V}} \circ \tilde{\alpha}$ is a lift of $p_{\mathcal{C}} \circ \alpha$, we have $p_{\mathcal{V} \#}[\tilde{\alpha}]=1 \in \pi_{1}(N(\mathcal{V}), *)$. Hence, every open cover $\mathcal{U}$ of $\bar{X}$ is refined by an open cover $\mathcal{V}$ of $\bar{X}$ such that $p_{\mathcal{V} \#}[\tilde{\alpha}]=1 \in \pi_{1}(N(\mathcal{V}), *)$. Consequently, $\psi([\tilde{\alpha}])=1 \in \check{\pi}_{1}\left(\bar{X}, \tilde{x}_{0}\right)$, as claimed.

However, $[\tilde{\alpha}] \neq 1 \in \pi_{1}\left(\bar{X}, \tilde{x}_{0}\right)$, because $[\alpha] \neq 1 \in \pi_{1}\left(X, x_{0}\right)$. This contradicts Theorem 2,

Recall that a compact metric space of trivial shape is called cell-like. Recall also that a compact metric space is cell-like if and only if it contracts within every ANR that it is embedded in. Every cell-like subset $X$ of a 2-dimensional manifold $M^{2}$ is cellular in $M^{2}$, that is, $X$ has arbitrarily small neighborhoods in $M^{2}$, which are homeomorphic to open disks. While the path components of a cell-like planar continuum need not be contractible (e.g. for the Knaster continuum), we record as a corollary to Theorem 5 the following "folklore" fact:

Corollary 6 If $X$ is a cell-like subset of a 2-dimensional manifold $M^{2}$ and $x_{0} \in X$, then $\pi_{1}\left(X, x_{0}\right)=1$.

Proof Since the first shape homotopy group of a cell-like space is trivial, this follows directly from Theorem 5 .

Recall that a group $G$ is said to be locally free if each of its finitely generated subgroups is free. A group $G$ is called fully residually free if for every finite subset $\left\{g_{1}, g_{2}, \ldots, g_{n}\right\} \subseteq G \backslash\{1\}$ there is a homomorphism $\phi: G \rightarrow F$ to a free group $F$ such that $\phi\left(g_{i}\right) \neq 1$ for all $i \in\{1,2, \ldots, n\}$. Finally, $G$ is said to be residually finite if for every $g \in G \backslash\{1\}$ there is a homomorphism $\phi: G \rightarrow H$ to a finite group $H$ such that $\phi(g) \neq 1$.

Some of the algebraic implications of Theorem 5 are collected in the following:

Corollary 7 Let $X$ be a proper compact subset of a 2-dimensional manifold $M^{2}$ and $x_{0} \in X$. Then $\pi_{1}\left(X, x_{0}\right)$ is isomorphic to a subgroup of the limit of an inverse sequence of finitely generated free groups. In particular, $\pi_{1}\left(X, x_{0}\right)$ is locally free, fully residually free and residually finite. 
Proof Notice that we can express $\left(X, x_{0}\right)$ as the intersection of a nested sequence $\left(X_{i}, x_{0}\right)$ of compact 2-dimensional PL manifold neighborhoods of $X$ in $M^{2}$, with $\partial X_{i} \neq \emptyset$ for all $i$. Since each of the groups $\pi_{1}\left(X_{i}, x_{0}\right)$ is finitely generated and free, it follows from Theorem [5 and Remark 1 that $\pi_{1}\left(X, x_{0}\right)$ is isomorphic to a subgroup of the limit of an inverse sequence of finitely generated free groups.

A proof of the fact that every finitely generated subgroup of the limit of an inverse sequence of finitely generated free groups is free can be found in 6 , Theorem 1]. Using the projection maps of this inverse limit to its free terms, we see that $\pi_{1}\left(X, x_{0}\right)$ is fully residually free. The first proof of the fact that free groups are residually finite was given by Schreier [3. p. 97]. (This proof can also be found in [4, p. 11].) It follows that $\pi_{1}\left(X, x_{0}\right)$ is residually finite, since it is fully residually free.

Following [1, Definition 5.2], we call a space $X$ homotopically Hausdorff at the point $x_{0} \in X$, if for every $g \in \pi_{1}\left(X, x_{0}\right) \backslash\{1\}$ there is an open set $U \subseteq X$ with $x_{0} \in U$ such that there is no loop $\alpha:\left(S^{1}, *\right) \rightarrow\left(U, x_{0}\right)$ with $[\alpha]=g$. If $X$ is homotopically Hausdorff at every one of its points, then $X$ is said to be homotopically Hausdorff. In [5, Theorem 3.4] it is shown that all planar sets are homotopically Hausdorff. Theorem 5 above is a strengthening of this statement, because we have the following:

Proposition 8 Let $X$ be a topological space and $x_{0} \in X$. If the natural homomorphism $\varphi: \pi_{1}\left(X, x_{0}\right) \rightarrow \check{\pi}_{1}\left(X, x_{0}\right)$ is injective, then $X$ is homotopically Hausdorff at $x_{0}$. However, the converse need not hold.

Proof An element $g \in \pi_{1}\left(X, x_{0}\right)$, witnessing a violation of the definition of homotopically Hausdorff at $x_{0}$, will be mapped to the trivial element of the fundamental group of the pointed nerve of every open cover of $X$, contradicting the injectivity of $\varphi: \pi_{1}\left(X, x_{0}\right) \rightarrow \check{\pi}_{1}\left(X, x_{0}\right)$. Example 9 below exhibits a space which is homotopically Hausdorff and for which $\varphi$ is not injective.

Example 9 This space was previously studied in [9]. Let

$$
Y=\left\{(x, y, z) \in \mathbb{R}^{3} \mid z=0,0<x \leqslant 1, y=\sin (1 / x)\right\} \cup(\{0\} \times[-1,1] \times\{0\})
$$

be the "topologist's sine curve". Define $Y_{i}=Y \cup([0,1 / i] \times[-1,1] \times\{0\})$. Let $X$ and $X_{i}$ be the subsets of $\mathbb{R}^{3}$ obtained by revolving $Y$ and $Y_{i}$ about the $y$-axis, respectively, and let $f_{i, i+1}: X_{i+1} \hookrightarrow X_{i}$ be inclusion. Then $X$ is the limit of the inverse sequence $\left(X_{i}, f_{i, i+1}\right)$. If we take $x_{0}=(1, \sin 1,0)$, then $\pi_{1}\left(X, x_{0}\right)$ is infinite cyclic, while $\check{\pi}_{1}\left(X, x_{0}\right)$ is trivial. We observe that $\varphi: \pi_{1}\left(X, x_{0}\right) \rightarrow \check{\pi}_{1}\left(X, x_{0}\right)$ is not injective, while $X$ is homotopically Hausdorff. 
Combining Theorem $[5$ with Proposition 8 also yields a proof of the following:

Corollary 10 Every subset $X$ of a closed surface is homotopically Hausdorff.

\section{Some special properties of subsets of the plane}

Definition 11 Consider a map $\alpha: S^{1} \rightarrow \mathbb{R}^{2}$ and let $O$ be the unbounded (path) component of $\mathbb{R}^{2} \backslash \alpha\left(S^{1}\right)$. We will call $\mathbb{R}^{2} \backslash O$ the inner region of $\alpha$ and denote it by $I(\alpha)$.

The inner region $I(\alpha)$ of any map $\alpha: S^{1} \rightarrow \mathbb{R}^{2}$ is a Peano continuum. This follows from the following lemma.

Lemma 12 Let $X \subseteq Y$ be two Peano continua, i.e., two compact, connected and locally (path) connected metric spaces. Let $\mathcal{C}$ be the collection of (path) components of $Y \backslash X$ and $\mathcal{C}^{\prime} \subseteq \mathcal{C}$. Then $Z=X \cup \cup \mathcal{C}^{\prime}$ is also a Peano continuum.

Proof By assumption, $(Y, d)$ is a metric space. We will verify that $Z$ is (i) compact, (ii) connected, and (iii) locally path connected.

(i) Since each element of $\mathcal{C}$ is open in $Y$, then $Z=Y \backslash \bigcup\left[\mathcal{C} \backslash \mathcal{C}^{\prime}\right]$ is a closed subset of the compact space $Y$. Hence $Z$ is compact.

(ii) Since $X$ is path connected, it will suffice to show that for every $z \in U \in \mathcal{C}^{\prime}$ there is an $x \in X$ and a path $p:[0, t] \rightarrow Z$ from $p(0)=z$ to $p(t)=x$. To this end, pick any $y \in X$ and choose a path $q:[0,1] \rightarrow Y$ from $q(0)=z$ to $q(1)=y$. Let $t=\min \left[q^{-1}(Y \backslash U)\right]$. Then $p=\left.q\right|_{[0, t]}$ and $x=p(t)$ will work.

(iii) Let $x \in Z$. Note that every element of $\mathcal{C}^{\prime}$ is an open subset of the locally path connected space $Y$. Hence, in order to show that $Z$ is locally path connected at $x$, we may assume that $x \in X$. Let $\epsilon>0$ be given. We will find a $\delta>0$ such that every point of $Z \cap N(x, \delta)$ can be joined to $x$ by a path that lies in $Z \cap N(x, \epsilon)$. Here, we adopt the usual notation $N(y, \eta)=\{z \in Y \mid d(z, y)<$ $\eta\}$. Since $X$ is locally path connected, we can choose an $\eta \in(0, \epsilon)$ such that every point of $X \cap N(x, \eta)$ can be joined to $x$ by a path that lies in $X \cap N(x, \epsilon)$. Since $Y$ is also locally path connected, we can choose a $\delta \in(0, \eta)$ such that every point of $N(x, \delta)$ can be joined to $x$ by a path that lies in $N(x, \eta)$.

Now, let $y \in Z \cap N(x, \delta)$. We may assume that $y \notin X$. Choose $U \in \mathcal{C}^{\prime}$ with $y \in U$. Choose a path $p:[0,1] \rightarrow N(x, \eta)$ from $p(0)=y$ to $p(1)=x$. Let $t=\min \left[p^{-1}(Y \backslash U)\right]$. Then $\left.p\right|_{[0, t]}$ is a path from $y$ to $p(t) \in X \cap N(x, \eta)$ that lies in $U \cap N(x, \eta)$, and therefore lies in $Z \cap N(x, \epsilon)$. Since there is another path in $X \cap N(x, \epsilon)$ from $p(t)$ to $x$, we are done. 
Lemma 13 Let any set $X \subseteq \mathbb{R}^{2}$ and any map $\alpha: S^{1} \rightarrow X$ be given. Let $I(\alpha)$ be as in Definition [1] If $\alpha: S^{1} \rightarrow X$ is null-homotopic, then so is $\alpha: S^{1} \rightarrow X \cap I(\alpha)$.

Proof Let us identify $\mathbb{R}^{2}$ with $\mathbb{S}^{2} \backslash\{\infty\}$ by means of stereographic projection and write $I(\alpha)=\mathbb{S}^{2} \backslash U$, where $U$ is the (path) component of $\infty$ in $\mathbb{S}^{2} \backslash \alpha\left(S^{1}\right)$. Observe that $U$ is a simply connected open subset of $\mathbb{S}^{2}$. (For if there were a homotopically non-trivial loop in $U$, which we might as well assume to be piecewise linear, it would have to contain a simple closed curve which is nontrivial in $U$. However, such a non-trivial simple closed curve of $U$ would have to separate points of $I(\alpha)$. This would contradict the path connectedness of $I(\alpha)$.) Moreover, we may assume that the complement of $U$ in $\mathbb{S}^{2}$, namely $I(\alpha)$, contains more than one point. Hence, by the Riemann Mapping Theorem, there is a biholomorphic map $g: \mathbb{D} \rightarrow U$ whose domain is the open unit disk $\mathbb{D} \subseteq \mathbb{C}$. Since $I(\alpha)$ is locally path connected, there is a map $\bar{g}: \overline{\mathbb{D}} \rightarrow \mathbb{S}^{2}$, whose domain is the closed unit disk $\overline{\mathbb{D}}$, such that $\left.\bar{g}\right|_{\mathbb{D}}=g$ and $\bar{g}(\partial \overline{\mathbb{D}})=$ bdy $U$ [17, Theorem 2.1]. Put $d=g^{-1}(\infty) \in \mathbb{D}$ and choose a continuous retraction $r: \overline{\mathbb{D}} \backslash\{d\} \rightarrow \partial \overline{\mathbb{D}}$ with $\left.r\right|_{\partial \overline{\mathbb{D}}}=i d$. Since $\left.\bar{g}\right|_{\overline{\mathbb{D}} \backslash\{d\}}: \overline{\mathbb{D}} \backslash\{d\} \rightarrow[\operatorname{cl} U] \backslash\{\infty\}$ is a quotient map, $r$ induces a continuous retraction $R:[\operatorname{cl} U] \backslash\{\infty\} \rightarrow$ bdy $U \subseteq I(\alpha)$ such that $\left.R\right|_{\text {bdy } U}=i d$, which we can extend to a continuous retraction $R: \mathbb{R}^{2} \rightarrow I(\alpha)$ by defining $R(x)=x$ for all $x \in I(\alpha)$.

Now, if $H: S^{1} \times[0,1] \rightarrow X$ is a homotopy contracting $\alpha$ to a point within $X$, that is, $H(s, 0)=\alpha(s)$ for all $s \in S^{1}$ and $H\left(S^{1}, 1\right)$ is a singleton, then the homotopy $R \circ H: S^{1} \times[0,1] \rightarrow I(\alpha)$ contracts $\alpha$ to a point within $X \cap I(\alpha)$. Indeed, if $H(s, t) \in I(\alpha)$, then $R(H(s, t))=H(s, t) \in X$, and if $H(s, t) \in U$, then we have $R(H(s, t)) \in$ bdy $U \subseteq \alpha\left(S^{1}\right) \subseteq X$, also.

Definition 14 A sequence $\left(C_{i}\right)$ of subsets of a metric space is called a nullsequence if for every $\epsilon>0, \operatorname{diam}\left(C_{i}\right) \leqslant \epsilon$ for all but finitely many $i$.

The following theorem is based on 19 .

Theorem 15 Let $Y \subseteq \mathbb{R}^{2}$ be a Peano continuum. Fix any $x_{0} \in Y$. List the bounded (path) components of $\mathbb{R}^{2} \backslash Y$ in a (possibly finite) pairwise disjoint sequence $C_{1}, C_{2}, C_{3}, \ldots$ From each $C_{i}$ let one point $z_{i}$ be given. Then there is a sequence $L_{1}, L_{2}, L_{3}, \ldots$ of subsets of $\mathbb{R}^{2}$ such that $L_{1}$ is a closed disk and each $L_{i+1}$ is obtained from $L_{i}$ by removing the interior of a closed disk $D_{i}$ in the interior of $L_{i}$ with the following properties: $z_{i} \in \operatorname{int} D_{i}$ for all $i$; $\left(D_{i}\right)$ forms a null-sequence; $Y \subseteq L_{i}$ for all $i$ and, setting $L=\bigcap_{i} L_{i}$, the inclusion $\left(Y, x_{0}\right) \hookrightarrow\left(L, x_{0}\right)$ is a pointed homotopy equivalence. 
The proof of Theorem 15 begins with the following lemma.

Lemma 16 Let $Y \subseteq \mathbb{R}^{2}$ be a Peano continuum. Then any pairwise distinct sequence of bounded (path) components of $\mathbb{R}^{2} \backslash Y$ is a null-sequence.

Proof Suppose, to the contrary, that there is an $\epsilon>0$ and infinitely many distinct bounded components $C_{1}, C_{2}, C_{3}, \ldots$ of $\mathbb{R}^{2} \backslash Y$ with $\operatorname{diam}\left(C_{i}\right)>\epsilon$ for all $i$. For each $i \in \mathbb{N}$ choose points $x_{i}=\left(a_{i}, b_{i}\right) \in C_{i}$ and $y_{i}=\left(c_{i}, d_{i}\right) \in C_{i}$ with $d\left(x_{i}, y_{i}\right)>\epsilon$. Since $\bigcup_{i} C_{i}$ is bounded, so are the sequences $\left(x_{i}\right)$ and $\left(y_{i}\right)$. Passing to subsequences, we may therefore assume that there are $x, y \in \mathbb{R}^{2}$ such that $\left(x_{i}\right)$ converges to $x$ and $\left(y_{i}\right)$ converges to $y$. Then $d(x, y) \geqslant \epsilon$. Changing our coordinate system, if necessary, we may assume that $x=(-2,0)$ and $y=(2,0)$. For $j \in\{-1,0,1\}$, consider the three vertical lines $L_{j}=\{(j, r) \mid r \in \mathbb{R}\}$. Discarding finitely many indices, we may assume that $a_{i}<-1$ and $c_{i}>1$ for all $i \in \mathbb{N}$. Since every $C_{i}$ is a path connected open subset of the plane containing points to the left of $L_{-1}$ as well as points to the right of $L_{1}$, we may choose for each $i \in \mathbb{N}$ a polygonal arc $\alpha_{i}$ in $C_{i}$ such that $\alpha_{i} \cap L_{-1}=\left\{s_{i}\right\}, \alpha_{i} \cap L_{1}=\left\{t_{i}\right\}$, $\partial \alpha_{i}=\left\{s_{i}, t_{i}\right\}$, and $\alpha_{i}$ is in general position with respect to $L_{0}$. Passing to another subsequence, we may assume that the sequence $\left(s_{i}\right)$ is monotone, say (strictly) increasing. Then the sequence $\left(t_{i}\right)$ also increases (strictly), because the arcs $\alpha_{1}, \alpha_{2}, \alpha_{3}, \ldots$ are pairwise disjoint. For each fixed $i \in \mathbb{N}$, consider the closed disk $D_{i}$, bounded by the simple closed curve consisting of $\alpha_{i}, \alpha_{i+1}$, and the two vertical closed intervals $\left[s_{i}, s_{i+1}\right]$ and $\left[t_{i}, t_{i+1}\right]$. Since $L_{0}$ separates $\left[s_{i}, s_{i+1}\right]$ from $\left[t_{i}, t_{i+1}\right]$, there is a vertical interval $\left[u_{i}, v_{i}\right] \subseteq L_{0} \cap D_{i}$ such that $u_{i}$ lies in one of the two $\operatorname{arcs} \alpha_{i}$ or $\alpha_{i+1}$ and $v_{i}$ lies in the other arc. Now, $\left[u_{i}, v_{i}\right]$ is an arc connecting the two distinct complementary components $C_{i}$ and $C_{i+1}$ of $Y$ in $\mathbb{R}^{2}$. Hence, we can choose a point $w_{i} \in\left(u_{i}, v_{i}\right) \cap Y$. Since $\left(w_{i}\right)$ is a bounded sequence in $L_{0}$, we may pass to a subsequence and assume that $\left(w_{i}\right)$ converges to some $w \in L_{0}$. In fact, since $Y$ is closed and each $w_{i} \in Y$, we must have $w \in Y$. By construction, $D_{i} \cap D_{i+1}=\alpha_{i+1}$ and $D_{i} \cap D_{j}=\emptyset$ for all $j>i+1$. Also, the points $w_{i}$ lie in the pairwise disjoint interiors of the disks $D_{i}$. Consequently, $w \notin D_{i}$ for all $i \in \mathbb{N}$. We conclude that every path in $Y$ connecting $w$ to $w_{i}$ must meet either $\left[s_{i}, s_{i+1}\right] \subseteq L_{-1}$ or $\left[t_{i}, t_{i+1}\right] \subseteq L_{1}$, because $\alpha_{i} \cap Y=\emptyset$ for all $i \in \mathbb{N}$. Consequently, $Y$ is not locally path connected at $w$, which is a contradiction.

Proof of Theorem 15] As in the proof of Lemma [13, we identify $\mathbb{R}^{2}$ with $\mathbb{S}^{2} \backslash\{\infty\}$. We put $z_{0}=\infty$ and let $C_{0}$ denote the (path) component of $z_{0}$ in $\mathbb{S}^{2} \backslash Y$. Note that $\mathbb{S}^{2} \backslash C_{0}=Y \cup \bigcup_{i \geqslant 1} C_{i}$ is a Peano continuum, by Lemma 12. Therefore, as in the proof of Lemma 13, we obtain a biholomorphic 
map $g:(\mathbb{D}, *) \rightarrow\left(C_{0}, z_{0}\right)$ with continuous extension $\bar{g}: \overline{\mathbb{D}} \rightarrow \mathbb{S}^{2}$ such that $\bar{g}(\partial \mathbb{D})=$ bdy $C_{0}$. Choose a closed disk $D$ with $* \in$ int $D \subseteq D \subseteq \mathbb{D}$ and put $D_{0}=g(D)$. Then $z_{0} \in \operatorname{int} D_{0} \subseteq D_{0} \subseteq C_{0}$. Choose a deformation retraction $H$ of $\overline{\mathbb{D}} \backslash$ int $D$ onto $\partial \mathbb{D}$, that is, choose a map $H:(\overline{\mathbb{D}} \backslash$ int $D) \times[0,1] \rightarrow \overline{\mathbb{D}} \backslash$ int $D$ with $H(x, 0)=x$ for all $x \in \overline{\mathbb{D}} \backslash$ int $D, H(x, 1) \in \partial \mathbb{D}$ for all $x \in \overline{\mathbb{D}} \backslash$ int $D$, and $H(x, t)=x$ for all $x \in \partial \mathbb{D}$ and all $t \in[0,1]$. Since

$$
\left.\bar{g}\right|_{\overline{\mathbb{D}} \backslash \operatorname{int} D} \times i d:(\overline{\mathbb{D}} \backslash \operatorname{int} D) \times[0,1] \rightarrow\left(\operatorname{cl} C_{0} \backslash \operatorname{int} D_{0}\right) \times[0,1]
$$

is a quotient map, the deformation retraction $H$ induces a deformation retraction $F_{0}$ of cl $C_{0} \backslash$ int $D_{0}$ onto bdy $C_{0}$.

If we choose closed disks $D_{i}$ with $z_{i} \in \operatorname{int} D_{i} \subseteq D_{i} \subseteq C_{i}$ for $i \geqslant 1$ in the same way, we obtain deformation retractions $F_{i}$ of $\mathrm{cl} C_{i} \backslash$ int $D_{i}$ onto bdy $C_{i}$ for all $i \geqslant 0$.

Define $L_{1}=\mathbb{S}^{2} \backslash \operatorname{int} D_{0}, L_{i+1}=L_{i} \backslash \operatorname{int} D_{i}$ and $L=\bigcap_{i} L_{i}$. Since $F_{i}(x, t)=x$ whenever $x \in$ bdy $C_{i}$ and since $C_{0}, C_{1}, C_{2}, \ldots$ are pairwise disjoint, we may combine these deformation retractions into one function $F: L \times[0,1] \rightarrow L$ by defining $F(x, t)=F_{i}(x, t)$ if $x \in \operatorname{cl} C_{i}$ for some $i \geqslant 0$, and $F(x, t)=x$ otherwise. Then $F(x, 0)=x$ for all $x \in L, F(x, 1) \in Y$ for all $x \in L$, and $F(x, t)=x$ for all $x \in Y$ and all $t \in[0,1]$. In order to complete the proof of the theorem, we only need to establish continuity of $F$.

Let $(x, t) \in L \times[0,1]$ be given. Since each $F_{i}$ is continuous, we may assume that $x$ is not contained in any of the open sets $C_{i}$. Then $F(x, t)=x$. Let $\epsilon>0$ be given. By Lemma [16] we may choose $N \in \mathbb{N}$ such that $\operatorname{diam}\left(\operatorname{cl} C_{i}\right)=$ $\operatorname{diam}\left(C_{i}\right) \leqslant \epsilon / 2$ for all $i>N$. Define $I=\left\{i \in\{0,1,2, \ldots, N\} \mid x \in \operatorname{cl} C_{i}\right\}$. Choose $\delta>0$ such that (i) $d(F(x, t), F(y, s))=d\left(F_{i}(x, t), F_{i}(y, s)\right)<\epsilon$ whenever $(y, s) \in L \times[0,1]$ with $y \in \operatorname{cl} C_{i}$ for some $i \in I, d(x, y)<\delta$ and $|s-t|<\delta$; (ii) $y \notin \mathrm{cl} C_{i}$ whenever $i \in\{0,1,2, \ldots, N\} \backslash I$ and $d(x, y)<\delta$; and (iii) $\delta<\epsilon / 2$. Let $(y, s) \in L \times[0,1]$ with $d(x, y)<\delta$ and $|s-t|<\delta$ be given. If $y \notin \mathrm{cl} C_{i}$ for any $i \geqslant 0$, then $F(y, s)=y$ so that $d(F(x, t), F(y, s))=d(x, y)<\delta<\epsilon / 2<\epsilon$, by (iii). We may therefore assume that $y \in \operatorname{cl} C_{i}$ for some $i \geqslant 0$. If $i>N$, then $d(F(x, t), F(y, s))=d(x, F(y, s)) \leqslant d(x, y)+d(y, F(y, s))<\delta+\epsilon / 2<\epsilon / 2+\epsilon / 2$, because both $y$ and $F(y, s)$ are contained in $\operatorname{cl} C_{i}$ and $\operatorname{diam}\left(\operatorname{cl} C_{i}\right) \leqslant \epsilon / 2$. Hence, we may assume that $0 \leqslant i \leqslant N$. Since $d(x, y)<\delta$ and $y \in \operatorname{cl} C_{i}$, then $i \in I$ by (ii). Consequently, $d(F(x, t), F(y, s))<\epsilon$ by (i).

The following theorem can be extracted from [19, where its development is a sideline of a comprehensive study of higher dimensional homotopy groups of planar sets. However, it is not explicitly stated in [19] and its proof is scattered 
throughout the lengthy document. We will therefore sketch a proof in the appendix.

Theorem 17 Let $L_{1}, L_{2}, L_{3}, \ldots$ be a sequence of subsets of $\mathbb{R}^{2}$ such that $L_{1}$ is a closed disk and each $L_{i+1}$ is obtained from $L_{i}$ by removing the interior of a closed disk $D_{i}$ in the interior of $L_{i}$ such that $\left(D_{i}\right)$ forms a null-sequence. If $x_{0} \in L=\bigcap_{i} L_{i}$, then the natural homomorphism $\varphi: \pi_{1}\left(L, x_{0}\right) \rightarrow \check{\pi}_{1}\left(L, x_{0}\right)$ is injective.

Remark 18 While it is known [8, Corollary 1.2] that $\varphi: \pi_{1}\left(X, x_{0}\right) \rightarrow \check{\pi}\left(X, x_{0}\right)$ is injective for 1-dimensional continua $X$, a space $L$ as described in Theorem 17 need not be homotopy equivalent to a 1-dimensional metric space (see [2, §5] and [13]).

\section{Appendix}

The purpose of this appendix is to sketch a proof of Theorem 17

Step 0 Reduction of Theorem [17 to a theorem about Sierpiński-like spaces.

For $i \in \mathbb{N}$, consider the collection $\mathcal{Q}_{i}^{* *}$ of all subsquares of $[0,1] \times[0,1]$ of the form $\left[\frac{2 k-1}{3^{i}}, \frac{2 k}{3^{i}}\right] \times\left[\frac{2 m-1}{3^{i}}, \frac{2 m}{3^{i}}\right]$ with $k, m \in \mathbb{N}$. Let $\mathcal{Q}_{i}^{*}$ be the collection of all elements of $\mathcal{Q}_{i}^{* *}$ which are not contained in any element of $\mathcal{Q}_{s}^{* *}$ with $s<i$. Figure 1 (left) depicts the union of the collections $\mathcal{Q}_{1}^{*}, \mathcal{Q}_{2}^{*}$ and $\mathcal{Q}_{3}^{*}$ in $[0,1] \times[0,1]$. Let $S$ be any space obtained from $[0,1] \times[0,1]$ by removing all, some, or none of the interiors of the squares in $\bigcup_{i} \mathcal{Q}_{i}^{*}$. Specifically, for each $i \in \mathbb{N}$, choose any subset $\mathcal{Q}_{i} \subseteq \mathcal{Q}_{i}^{*}$. Inductively, define $S_{0}=[0,1] \times[0,1]$ and let $S_{i+1}$ be obtained from $S_{i}$ by removing the interiors of the elements of $\mathcal{Q}_{i}$, which we shall informally refer to as holes. Then $S=\bigcap_{i} S_{i}$ will be called a Sierpiński-like space with defining sequence $\left(S_{i}\right)$.

Note that if $\mathcal{Q}_{i}=\mathcal{Q}_{i}^{*}$ for all $i$, the resulting space $S$, which we will denote by $S^{*}$, is homeomorphic to the Sierpiński carpet. This follows from the topological characterization of the Sierpiński carpet given in [18, Theorems 3 and 4]: it is the unique planar 1-dimensional Peano continuum without local cut points. It also follows that if $L,\left(L_{i}\right)$ and $\left(D_{i}\right)$ are as in Theorem 17, then $L$ is homeomorphic to some Sierpiński-like space $S$. Indeed, we may extend the sequence $\left(D_{i}\right)$ to a dense null-sequence of pairwise disjoint disks $\left(D_{i}^{*}\right)$ in the interior of $L_{1}$. It follows from elementary considerations that removing the 

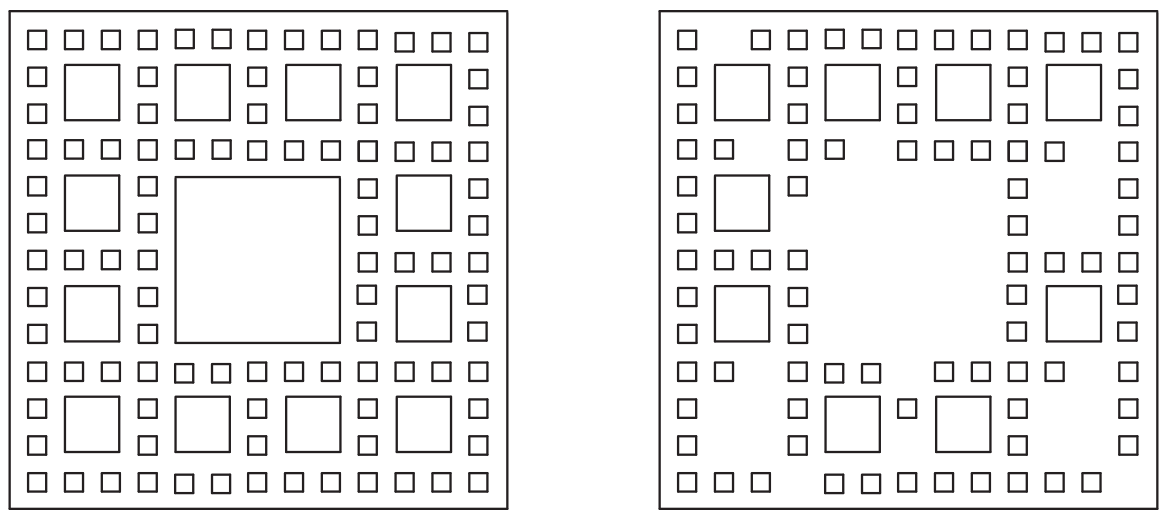

Figure 1: A Sierpiński-like pattern; complete or incomplete

interiors of all $D_{i}^{*}$ from $L_{1}$ leaves us with a planar 1-dimensional Peano continuum $L^{*}$ without local cutpoints, which consequently is homeomorphic to $S^{*}$. Observe that the boundary curves of the disks whose interiors are removed in either version of the Sierpiński carpet, $L^{*}$ or $S^{*}$, are characterized as the unique non-separating simple closed curves. Therefore, since $L$ is obtained by removing the interiors of only some of the disks $D_{i}^{*}$ from $L_{1}$, namely those of the disks $D_{i}$, the homeomorphism between $L^{*}$ and $S^{*}$ can be extended to a homeomorphism of $L$ onto $S$.

It therefore suffices to prove the following result.

Theorem Let $S$ be a Sierpiński-like space with defining sequence $\left(S_{i}\right)$ and $\alpha: S^{1} \rightarrow S$ a loop which is null-homotopic in $S_{i}$ for every $i \in \mathbb{N}$. Then $\alpha$ is null-homotopic in $S$.

We will call the closure of a component of $S_{i} \cap\left([0,1] \times\left(\frac{2 m-1}{3^{i}}, \frac{2 m}{3^{i}}\right)\right)$ a horizontal corridor of $S_{i}$ (on the $m$-th stratum) and denote the collection of all horizontal corridors of $S_{i}$ by $\mathcal{N}_{i}^{h}$. Analogously, we will call the closure of a component of $S_{i} \cap\left(\left(\frac{2 k-1}{3^{i}}, \frac{2 k}{3^{i}}\right) \times[0,1]\right)$ a vertical corridor of $S_{i}$ (on the $k$-th stratum) and denote the collection of all vertical corridors of $S_{i}$ by $\mathcal{N}_{i}^{v}$. If $[a, b] \times[c, d] \in \mathcal{N}_{i}^{h}$ is a horizontal corridor, we will refer to $[a, b] \times(c, d)$ as its inner region, to $[a, b] \times\{c\}$ and $[a, b] \times\{d\}$ as its boundary lines, and to $[a, b] \times\left\{\frac{c+d}{2}\right\}$ as its center line. Similar terminology will be applied to vertical corridors, but in the other direction.

Step 1 The idea is to encode how $\alpha$ traverses the network of corridors $\mathcal{N}_{i}=$ $\mathcal{N}_{i}^{h} \cup \mathcal{N}_{i}^{v}$ of every approximation level $S_{i}$, by associating to $\alpha$ a sequence of 
(cyclic) words $\omega=\left(\omega_{1}, \omega_{2}, \omega_{3}, \ldots\right)$, each of which is composed of letters that record which corridor was traversed in what direction, unless $\alpha$ does not cross any corridor of $\mathcal{N}_{i}$, in which case we record the empty word for $\omega_{i}$ on that level.

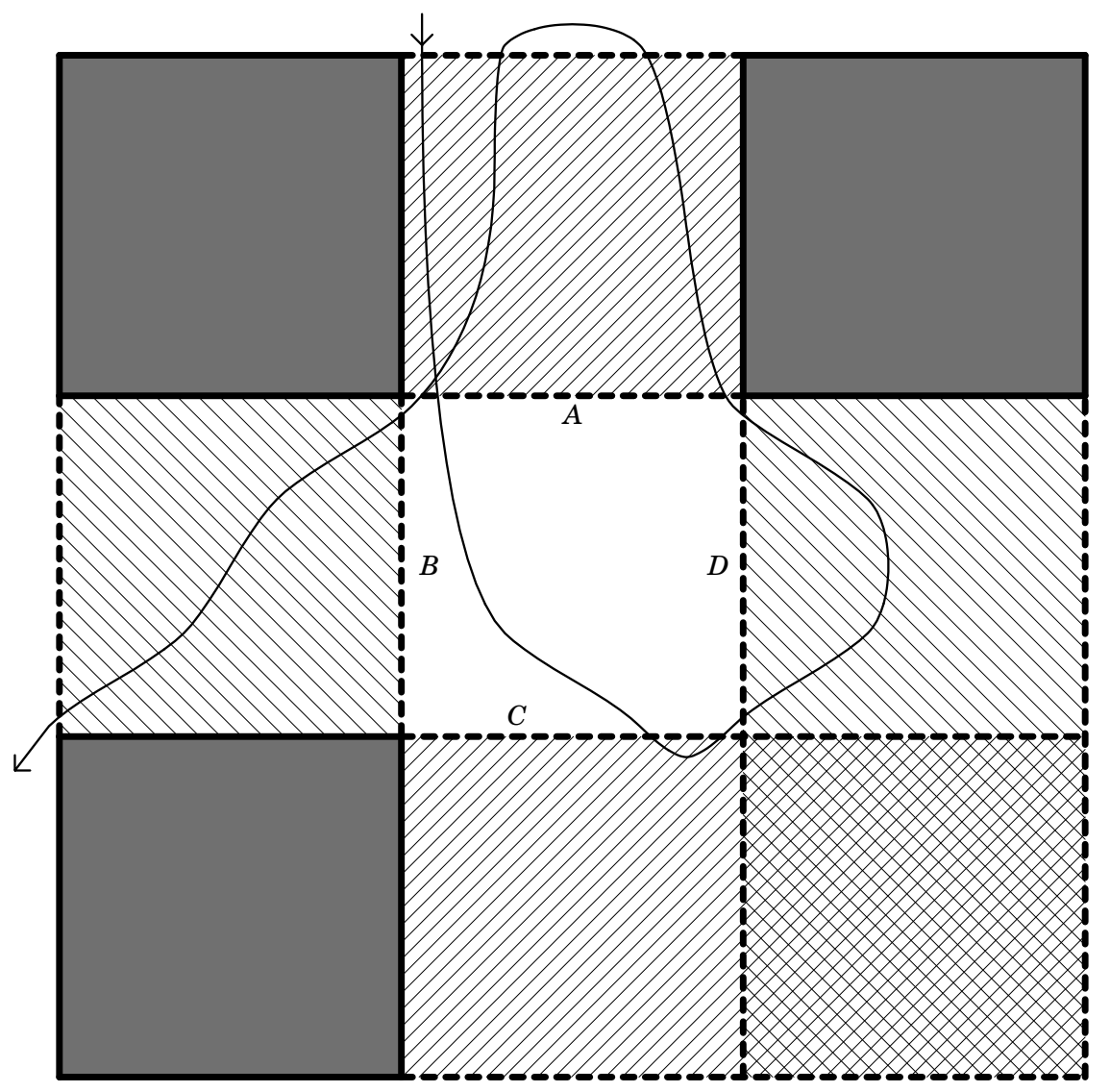

Figure 2: $\omega_{2}=A^{-1} A A^{-1} B^{-1} \ldots$

Figures 2 and 3 show a $\left[\frac{1}{9}, \frac{4}{9}\right] \times\left[\frac{1}{9}, \frac{4}{9}\right]$-detail of how such a word sequence might be formed through two levels of approximation: $\omega_{2}=A^{-1} A A^{-1} B^{-1} \cdots$ and $\omega_{3}=a_{1}^{-1} a_{3}^{-1} j^{-1} h d_{3} l d_{1}^{-1} a_{4} a_{2} e^{-1} a_{1}^{-1} a_{3}^{-1} b_{3}^{-1} j^{-1} b_{2}^{-1} \cdots$.

Before we discuss this coding procedure in more detail in Step 2 we would like to point to the heart of the problem at hand: consider the loop shown in Figure 4, which runs through a detail of Figure 3. This loop is null-homotopic in $S_{3}$ and Figure 4 depicts a possible cancellation diagram. Now, the hole on the left hand side of Figure 4 is an element of $\mathcal{Q}_{3}$. Therefore, if we consider the same loop in $S_{2}$ instead of $S_{3}$, drastically different cancellations appear 
plausible, including those that might not be feasible in $S_{3}$. However, at level $i=2$, we do not know whether or not such a hole might appear in $S_{3}$, or if instead a smaller version of it might appear in some $S_{i}$ with $i>3$, or even on which side of the figure it might appear. Therefore, a specific null-homotopy of $\alpha$ in $S_{i}$, which might have seemed like a natural choice at the time, will in general be useless for finding a null-homotopy of $\alpha$ in $S$, as it might not even yield a null-homotopy of $\alpha$ in $S_{j}$ if $j>i$. The word-calculus of [19] offers a solution to this complication.

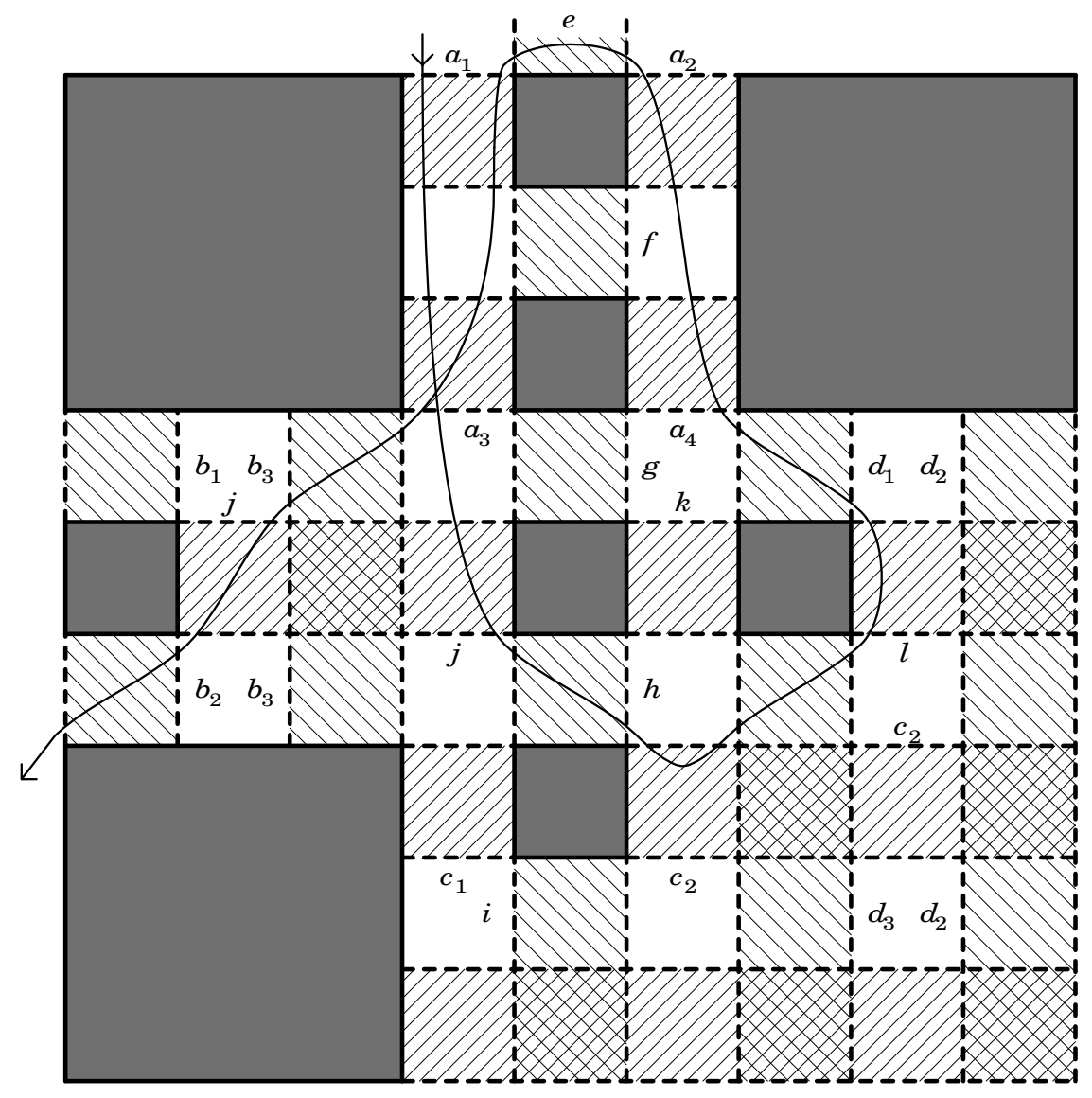

Figure 3: $\omega_{3}=a_{1}^{-1} a_{3}^{-1} j^{-1} h d_{3} l d_{1}^{-1} a_{4} a_{2} e^{-1} a_{1}^{-1} a_{3}^{-1} b_{3}^{-1} j^{-1} b_{2}^{-1} \ldots$

Step 2 We now describe a procedure for obtaining a suitable word sequence.

For each $i \in \mathbb{N}$, consider the collection $\mathcal{I}_{i}^{h}$ of all closed subintervals $[a, b]$ of $S^{1}$ for which $\alpha([a, b]) \subseteq C$ for some $C \in \mathcal{N}_{i}^{h}$, with $\alpha(a)$ on one boundary line of 
$C, \alpha(b)$ on the other boundary line of $C$, and such that $\alpha((a, b))$ lies in the interior region of $C$.

Then $\mathcal{I}_{i}^{h}$ consists of finitely many intervals with pairwise disjoint interiors. Moreover, we observe the following relationships between $\mathcal{I}_{i}^{h}$ and the corresponding collections $\mathcal{I}_{i-1}^{h}$ and $\mathcal{I}_{i+1}^{h}$ on its neighboring levels:

(R1) Consider any fixed horizontal stratum $E$ of level $(i-1)$ and its two substrata $E_{1}$ and $E_{2}$ of level $i$. If $[a, b]$ and $[c, d]$ are any intervals of $\mathcal{I}_{i}^{h}$ with $\alpha([a, b]) \subseteq E_{1}$ and $\alpha([c, d]) \subseteq E_{2}$, such that $[a, b]$ and $[c, d]$ are adjacent in $\mathcal{I}_{i}^{h}$ with this property, then $[a, d] \in \mathcal{I}_{i-1}^{h}$;

(R2) Conversely, for every $[a, d] \in \mathcal{I}_{i}^{h}$, there are $b, c \in[a, d]$ such that both $[a, b],[c, d] \in \mathcal{I}_{i+1}^{h}$.

Considering the vertical corridors instead, we arrive at a similar collection $\mathcal{I}_{i}^{v}$.

Form a (cyclic) ordered tuple $\left(\left[a_{1}, b_{1}\right],\left[a_{2}, b_{2}\right],\left[a_{3}, b_{3}\right], \ldots\right)$ by merging the elements of $\mathcal{I}_{i}^{h}$ and $\mathcal{I}_{i}^{v}$, while maintaining the relative natural order on $S^{1}$ within each collection; though allowing any relative order between two intersecting intervals $[a, b] \in \mathcal{I}_{i}^{h}$ and $\left[a^{\prime}, b^{\prime}\right] \in \mathcal{I}_{i}^{v}$ which are associated to crossing corridors. The word $\omega_{i}$ is then formed by replacing each interval $[a, b]$ of this sequence by the label of the corridor into which it maps under $\alpha$, along with an exponent indicating positive or negative direction.

Note that the word sequence $\left(\omega_{i}\right)$ contains all relevant information about the loop $\alpha$. Indeed, if we were to recreate $\alpha$ from the combinatorial data contained in the word sequence $\left(\omega_{i}\right)$ by forming polygonal loops $\alpha_{i}$ in $S_{i}$, which are in general position with respect to the boundary lines of $\mathcal{N}_{i}$ and which spell out the word $\omega_{i}$ by canonically traversing the prescribed corridors, then the sequence $\left(\alpha_{i}\right)$ would converge uniformly to a reparametrization of $\alpha$.

Step 3 Since $\alpha: S^{1} \rightarrow S_{i}$ is null-homotopic for every $i \in \mathbb{N}$, we can transform each word $\omega_{i}$ to the empty word by symbolic cancellations and transpositions, if we allow letters to commute whenever they correspond to crossing corridors.

Indeed, we can homotope $\alpha: S^{1} \rightarrow S_{i}$ to a polygonal loop $\alpha_{i}^{\prime}: S^{1} \rightarrow S_{i}$ which is in general position with respect to the center lines of all corridors of $\mathcal{N}_{i}$, while maintaining the sequence $\left(\left[a_{1}, b_{1}\right],\left[a_{2}, b_{2}\right],\left[a_{3}, b_{3}\right], \ldots\right)$ for the word $\omega_{i}$, and crossing the center lines only as often as needed to spell the word $\omega_{i}$. We then may contract $\alpha_{i}^{\prime}: S^{1} \rightarrow S_{i}$ with a homotopy $H_{i}^{\prime}: D^{2} \rightarrow S_{i}$ (also in general position) such that $\left.H_{i}^{\prime}\right|_{S^{1}}=\alpha_{i}^{\prime}$. Consider the preimage of the center lines of all corridors of $\mathcal{N}_{i}$ in $D^{2}$, under the homotopy $H_{i}^{\prime}$. It is the union of finitely many arcs, whose endpoints lie in $S^{1}$, and finitely many circles. Intersections among 
these arcs and circles can occur only if one of them maps into a horizontal center line and the other one into a vertical center line. We can eliminate the circles, one innermost circle at a time, by moving the homotopy across the appropriate center line. (Note that this can be done without disturbing the pairwise disjoint arc components of the preimage of the center lines of the other direction, which might span across these circles.) The endpoints of the remaining arcs lie in distinct elements of $\left(\left[a_{1}, b_{1}\right],\left[a_{2}, b_{2}\right],\left[a_{3}, b_{3}\right], \ldots\right)$, which correspond to letters of the same corridor but have opposite exponents. (To see why the exponents cannot agree, lift the entire null-homotopy to the universal cover of the at most twice punctured unit square; punctured by the hole(s) at the end of that corridor. An innermost mismatching arc would, when following the lift of $\alpha_{i}^{\prime}$ between its endpoints, contradict the fact that each component of the preimage of the center line separates this cover.) We therefore arrive at a pattern of arcs in $D^{2}$, which pairs up cancelling letters of $\omega_{i}$ across commuting letters as claimed.

In fact, we can shorten the word in every step of this transformation, so long as we allow for cancellations to take place across commuting variables, without formally performing any transpositions.

Step 4 If we repeat the above argument with the combined center lines of two adjacent levels $\mathcal{N}_{i}$ and $\mathcal{N}_{i-1}$, we see that every cancellation facility of $\omega_{i}$ thus obtained induces at least one cancellation facility of $\omega_{i-1}$, via the relationships $(R 1)$ and (R2) above. Moreover, we can select a reduction scheme for the entire word sequence $\left(\omega_{i}\right)$, which is coherent between levels.

To illustrate the idea, consider the cancellation diagram depicted in Figure 4 for the word $\omega_{3}=d_{3} d_{2} l d_{2}^{-1} l^{-1} d_{3}^{-1} k d_{1} l^{-1} d_{2} l d_{2}^{-1} d_{1}^{-1} k^{-1}$. It induces cancellations for the corresponding word $\omega_{2}=D D^{-1} D D^{-1}$ of the form $\left(D D^{-1}\right)\left(D D^{-1}\right)$, rather than the competing cancellations $D\left(D^{-1} D\right) D^{-1}$. In order to obtain a coherent reduction scheme for the entire word sequence $\left(\omega_{i}\right)$, we need to find cancellation diagrams for the individual words $\omega_{i}$, which induce each other, from one level to the previous one, in the above sense.

Having reduced this problem to a purely combinatorial issue, we can find a coherent scheme without much difficulty by considering the following underlying graph structure. We regard each specific cancellation of each word $\omega_{i}$ as one vertex of a graph whose (directed) edges indicate that a certain cancellation of $\omega_{i}$ induces a certain cancellation of $\omega_{i-1}$. One can now proceed as in König's classical method [14, VI. $\S 2$ Satz 6] for finding an infinite ray in a finitely branching infinite rooted tree: beginning at the root-vertex, select one of the finitely many adjacent vertices behind which there are infinitely many 

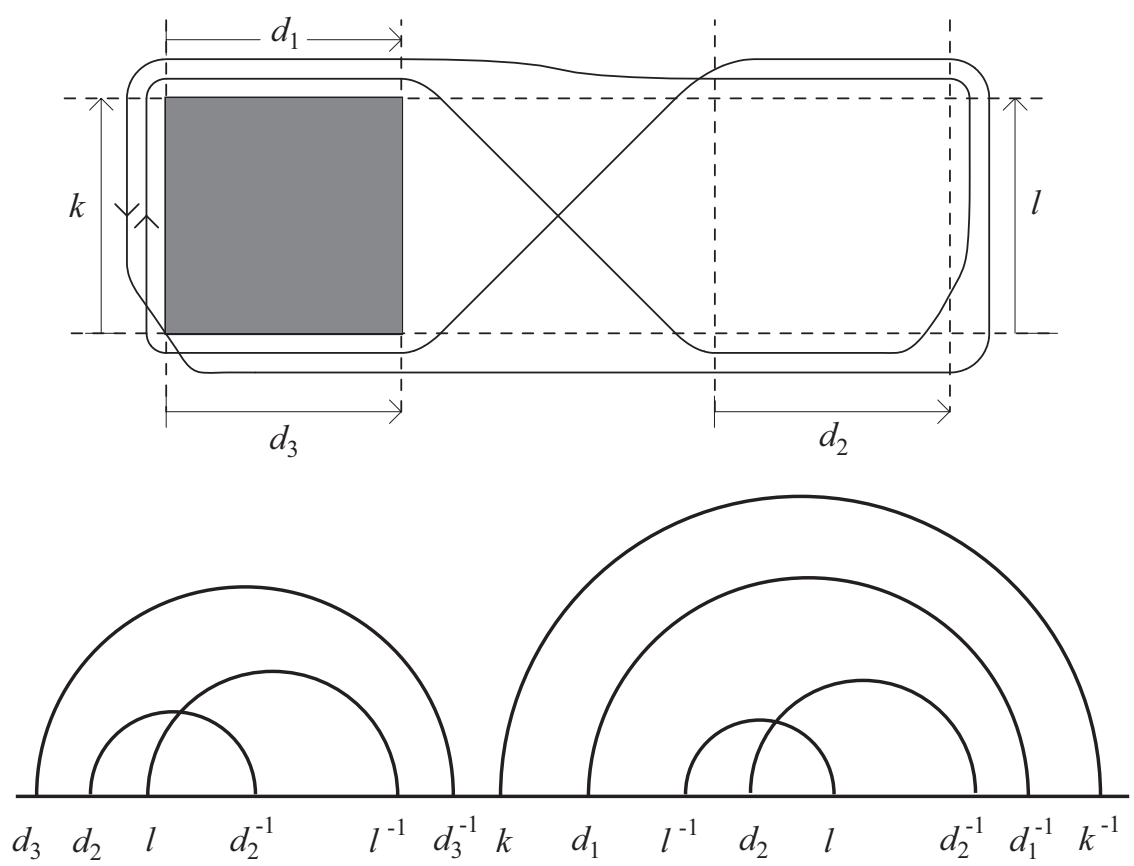

Figure 4: A transformation of the word $\omega_{3}$ to the empty word

vertices and construct the infinite ray iteratively by repeating this step. Put differently, the collection of cancellation patterns for the word sequence $\left(\omega_{i}\right)$ can be organized into an inverse system of nonempty finite sets. The inverse limit of this system is nonempty and any element of this limit provides a coherent set of cancellation patterns. (See [16, Prop. 2.9 - Cor. 2.11], where the latter argument is used in a similar situation.)

Step 5 The remainder of the proof consists of recording the resulting cancellation diagram for each level $i \in \mathbb{N}$ and turning it into a homotopy $H_{i}: D^{2} \rightarrow S_{i}$, such that the sequence $\left(H_{i}\right)$ converges uniformly to a homotopy $H: D^{2} \rightarrow S$ with $\left.H\right|_{S^{1}}=\alpha$.

The transformation information of Figure 4, for example, can be transcribed into the disk $D^{2}$, whose boundary $S^{1}$ is the domain of $\alpha$, featuring the intervals $\mathcal{I}_{i}^{h}$ and $\mathcal{I}_{i}^{v}$. The result is shown in Figure 5, where for each line of the cancellation diagram of Figure 4 we placed a band between the corresponding intervals of $S^{1}$. (Recall that an interval of $\mathcal{I}_{i}^{h}$ may overlap with an interval of $\mathcal{I}_{i}^{v}$.)

We thus obtain a sufficiently coherent sequence of cellulations of $D^{2}$ by bands. 
Because our transformations consist of cancellations, each such cellulation can be represented by (hyperbolic) straight line segments whose boundaries lie in $S^{1}$.

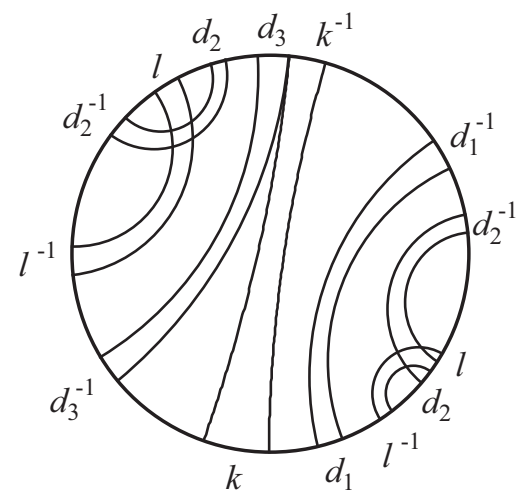

Figure 5: A cellulation of the disk induced by Figure 4

For a fixed $i \in \mathbb{N}$, we define $H_{i}: D^{2} \rightarrow S_{i}$ as follows. Begin by declaring that $\left.H_{i}\right|_{S^{1}}=\alpha$. On the two boundary lines of a band which is associated to a horizontal letter, we define the $y$-coordinates to be constant. Similarly, on the two boundary lines of a band which is associated to a vertical letter, we define the $x$-coordinates to be constant. Consequently, $H_{i}$ is defined on the zero-skeleton of our cellulation. The missing coordinate for a point on the one-skeleton is now obtained by a linear interpolation of the corresponding coordinates of the endpoints of this one-cell. By construction, when $H_{i}$ is restricted to the boundary of a two-cell, we obtain a null-homotopic path in $S_{i}$, yielding the missing values for $H_{i}$ in a canonical way. In order to describe how, we will refer to a square $\left[\frac{t}{3^{i}}, \frac{t+1}{3^{i}}\right] \times\left[\frac{s}{3^{i}}, \frac{s+1}{3^{i}}\right]$ of $S_{i}$ as being of type 0,1 , or 2 , according to how many corridors of $\mathcal{N}_{i}$ contain its interior. Since $H_{i}$ maps the boundary lines of each band into the corresponding corridor of $\mathcal{N}_{i}$, the crossing region of two bands, for example, can be mapped into one type- 2 square of $S_{i}$, which is the intersection of the corresponding corridors of $\mathcal{N}_{i}$. Similar arguments apply to the remaining types of two-cells: they can either be mapped into (i) regions formed by one square of type 1 along with at most two adjacent squares of type 2 ; or (ii) regions formed by one center square of type 0 along with at most eight adjacent squares of type 1 or 2. (Observe that the extended regions described in (i) and (ii) might be needed to accommodate the images of those two-cells, which intersect $\partial D^{2}$. Indeed, as shown in Figures 2 and 3. our loop might enter adjacent corridors without crossing them, thus not recording the corresponding letters in the word of this level and hence not 
contributing corresponding bands to the cellulation.)

Step 6 We claim that $\left\|H_{i}(z)-H_{i+1}(z)\right\| \leqslant\left(6 / 3^{i}\right)$ for every $i \in \mathbb{N}$ and for every $z \in D^{2}$. This will imply that the sequence $\left(H_{i}: D^{2} \rightarrow S_{i}\right)$ converges uniformly, and since $S=\bigcap_{i} S_{i}$, its limit will be a homotopy $H: D^{2} \rightarrow S$ with $\left.H\right|_{S^{1}}=\alpha$, thus completing the proof.

Let any $i \in \mathbb{N}$ and any $z \in D^{2}$ be given. Denoting $H_{i}(z)=\left(x_{i}, y_{i}\right)$ and $H_{i+1}(z)=\left(x_{i+1}, y_{i+1}\right)$, it suffices to argue that $\left|x_{i}-x_{i+1}\right|<\left(3 / 3^{i}\right)$ and $\left|y_{i}-y_{i+1}\right|<\left(3 / 3^{i}\right)$. We will only focus on the $y$-coordinates, since the argument for the $x$-coordinates is entirely analogous.

For the moment, consider only cellulation bands of level $i$, and only those which correspond to horizontal letters. Such bands do not intersect. Moreover, the (constant) $y$-values of $H_{i}$ on the boundary lines of these bands provide an estimate for the $y$-values of $H_{i}$ on the regions in between: by construction, $H_{i}$ never maps such regions across more than three adjacent horizontal sectors of the form $[0,1] \times\left[\frac{s}{3^{i}}, \frac{s+1}{3^{i}}\right]$ (see (i) and (ii) of Step [5), so that the $y$-values of $H_{i}$ on one such region never differ by more than $3 / 3^{i}$.

The same (even stronger) estimates hold for the $y$-values of $H_{i+1}$ in regions between boundary lines of cellulation bands of level $(i+1)$, which correspond to horizontal letters.

We claim that such estimates also hold across the two levels $i$ and $(i+1)$. For this to work, it is essential to have selected coherent cellulations between levels, because two bands from adjacent levels, both corresponding to horizontal letters, do not randomly cross. In fact, only one single, very benign, intersection phenomenon between their boundary lines is possible:

Consider a loop running through Figure 3, whose word $\omega_{3}=a_{3} a_{1} a_{1}^{-1} a_{1} a_{1}^{-1} a_{3}^{-1}$ cancels as $a_{3}\left(a_{1} a_{1}^{-1}\right)\left(a_{1} a_{1}^{-1}\right) a_{3}^{-1}$ and whose word $\omega_{2}=A A^{-1}$ cancels as $\left(A A^{-1}\right)$. Let $R$ be the band representing the cancellation of $A$ with $A^{-1}$ and let $r_{1}, r_{1}^{\prime}$ and $r_{3}$ be the bands representing the cancellations of $a_{1}$ with $a_{1}^{-1}$, $a_{1}$ with $a_{1}^{-1}$, and $a_{3}$ with $a_{3}^{-1}$, respectively. (See Figure 6) As described in (R1) and (R2) of Step 2 the bands $r_{1}$ and $r_{3}$ originate on either side of one end of the band $R$. While $r_{3}$, as one would expect, lies completely inside of $R$, sharing one boundary line with $R, r_{1}$ (as well as $r_{1}^{\prime}$ ) turns and leaves $R$ by crossing that boundary line of $R$ at which it originated.

This is indeed the only type of intersection of such bands, because their corresponding arcs in the preimage of the homotopy of Step 4, which correlates the cancellation diagrams of level $i$ and $(i+1)$, do not intersect at all. 


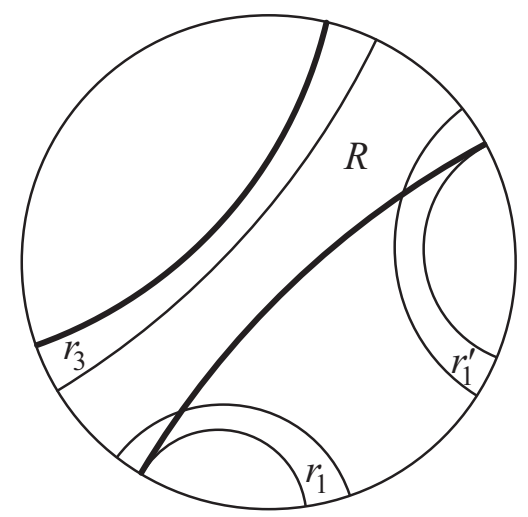

Figure 6: Cellulation bands of two adjacent levels - a worst case scenario

Since there are no further intersection phenomena, a straightforward region by region analysis of the common refinement of the two cellulations on level $i$ and level $(i+1)$, by bands corresponding to horizontal letters only, therefore yields $\left|y_{i}-y_{i+1}\right|<3 / 3^{i}$.

\section{References}

[1] J W Cannon, G R Conner, On the fundamental groups of one-dimensional spaces, preprint, Brigham Young University

[2] J W Cannon, G R Conner, A Zastrow, One-dimensional sets and planar sets are aspherical, Topology Appl. 120 (2002) 23-45 MathReview

[3] B Chandler, W Magnus, The history of combinatorial group theory, Studies in the History of Mathematics and Physical Sciences 9, Springer-Verlag, New York (1982) MathReview

[4] D E Cohen, Combinatorial group theory: a topological approach, London Mathematical Society Student Texts 14, Cambridge University Press, Cambridge (1989) MathReview

[5] G R Conner, J W Lamereaux, On the existence of universal covering spaces for metric spaces and subsets of the Euclidean plane, Fund. Math. 187 (2005) $95-110$

[6] ML Curtis, MK Fort, Jr, Singular homology of one-dimensional spaces, Ann. of Math. (2) 69 (1959) 309-313 MathReview

[7] K Eda, The fundamental groups of one-dimensional wild spaces and the Hawaiian earring, Proc. Amer. Math. Soc. 130 (2002) 1515-1522 MathReview 
[8] K Eda, K Kawamura, The fundamental groups of one-dimensional spaces, Topology Appl. 87 (1998) 163-172 MathReview

[9] H Fischer, C R Guilbault, On homotopy properties of certain Coxeter group boundaries, Proceedings of the 19th Annual Workshop in Geometric Topology, Calvin College, Grand Rapids, Michigan (2002) 39-46

[10] H Fischer, C R Guilbault, On the fundamental groups of trees of manifolds, Pacific J. Math. 221 (2005) 49-79

[11] HB Griffiths, The fundamental group of two spaces with a common point, Quart. J. Math. Oxford Ser. (2) 5 (1954) 175-190; Correction: ibid. 6 (1955) 154-155 MathReview

[12] H B Griffiths, A note on commutators in free products, Proc. Cambridge Philos. Soc. 50 (1954) 178-188 MathReview

[13] U H Karimov, D Repovš, W Rosicki, A Zastrow, On two-dimensional planar compacta not homotopically equivalent to any one-dimensional compactum, Topology Appl. 153 (2005) 284-293

[14] D König, Theorie der endlichen und unendlichen Graphen, Mathematik und ihre Anwendungen in Monographien und Lehrbüchern, Akademische Verlagsgesellschaft, Leipzig (1936)

[15] S Mardešić, J Segal, Shape theory, North-Holland Mathematical Library 26, North-Holland Publishing Co., Amsterdam (1982) MathReview

[16] J W Morgan, I Morrison, A van Kampen theorem for weak joins, Proc. London Math. Soc. (3) 53 (1986) 562-576 MathReview

[17] C Pommerenke, Boundary behaviour of conformal maps, Grundlehren series 299, Springer-Verlag, Berlin (1992) MathReview

[18] G T Whyburn, Topological characterization of the Sierpinski curve, Fund. Math. 45 (1958) 320-324 MathReview

[19] A Zastrow, Planar sets are aspherical, Thesis (Habilitationsschrift), RuhrUniversität Bochum (1997-98)

[20] A Zastrow, The second van-Kampen theorem for topological spaces, Topology Appl. 59 (1994) 201-232 MathReview

Department of Mathematical Sciences, Ball State University

Muncie, IN 47306, USA

and

Institute of Mathematics, University of Gdańsk

ul. Wita Stwosza 57, 80-952 Gdańsk, Poland

Email: fischer@math.bsu.edu and zastrow@math.univ.gda.pl

Received: 7 November 2005 